GALIANO. Flora de Andalucia Occidental 2: 257-258. Barcelona.

MARTÍNEZ, J. B. -1993- Rorippa Scop. In: S.

CASTROVIEJO et al., Flora Iberica 4: 106117. Madrid.

McNEILL, J. - 1980- Scilla L. In: T. G. TUTIN, V.

H. HEYWOOD, N. A. BURGES, D. M. MOORE,

D.H. VALENTINE, S.M. WALTERS \& D. A.

WEBB. Flora Europaea 5: 41-43. Cambridge

PEREIRA COUTINHO, A. X. -1939-Flora de

Portugal (plantas vasculares) disposta em chaves dicotómicas. $2^{\mathrm{a}}$ edición. Lisboa.

RICO, E., A. SÁNCHEZ y F. AMICH -1982-

Contribución a la flora del centro-oeste español.

Trab. Dept. Bot. Salamanca 10: 29-40.

RIVAS GODAY, S. -1964-Vegetación y Flórula de

la cuenca Extremeña del Guadiana. Madrid.
SILVESTRE, S. y P. MONSERRAT -1998- Rosa L. In. S. CASTROVIEJO. Flora Iberica 6: 143195. Madrid.

TALAVERA S. \& P. E. GIBBS - 1999- Teline Medik. In: S. CASTROVIEJO. Flora Iberica. 7(1): 141 148. Madrid.

Aceptado para su publicación en septiembre de 2002

Dirección de los autores. Consejería de Agricultura y Medio Ambiente. Dirección General de producción, Investigación y Formación Agraria. Avda. de Portugal, s/n. 06008, Mérida.

\title{
116. NOUVELLES CITATIONS POUR LA FLORE DE L'ARCHIPEL DES BERLENGAS (ESTREMADURA, PORTUGAL)
}

Cristina TAULEIGNE GOMES

Novas citações para a flora do arquipélago das Berlengas (Estremadura, Portugal)

Mots clé. Chorologie, îles, Reserva Natural das Berlengas, Portugal.

Palavras chave. Corologia, ilhas, Reserva Natural das Berlengas, Portugal.

La zone protégée Reserva Natural das Berlengas à laquelle appartiennent l'île de Berlenga et les îlots des Estelas, Medas, Farilhões et Forcadas est localisée à $10 \mathrm{~km}$ du littoral atlantique portugais $\left(39^{\circ} 24^{\prime} 49^{\prime \prime} \mathrm{N}\right.$ et $9^{\circ} 30^{\prime} 29^{\prime \prime}$ W). Le relief de Berlenga, l'île principale de l'archipel se caractérise par un plateau granitique érodé de $89 \mathrm{~m}$ d'altitude et
78.8 ha de surface et de nombreuses falaises abruptes.

Le climat est de type méditerranéen avec une température moyenne annuelle de $15.0^{\circ} \mathrm{C}$ et une hauteur annuelle des précipitations de $549.4 \mathrm{~mm}$. L'influence atlantique est manifeste avec une moyenne annuelle du taux d'humidité relative de l'air de $84 \%$ et une amplitude 
thermique entre les moyennes mensuelles de 7.1 ${ }^{\circ} \mathrm{C}$ - valeurs pour la période 1931-1960, station météorologique de Cabo Carvoeiro (Serviço Meteorológico Nacional, 1970).

Entre 1989 et 2001 un total de 128 espèces ont été observées sur l'archipel (Tauleigne Gomes \& Lefèbvre, submitted). La Flore est riche de plusieurs éléments endémiques de l'archipel : Armeria berlengensis Daveau, Herniaria berlengiana (Chaudhri) Franco inclus dans la Directive 92/43/CEE Annexe II, et Pulicaria microcephala Lange. Plusieurs espèces endémiques du littoral de la Péninsule Ibérique sont présentes : Echium rosulatum Lange, Scrophularia sublyrata Brot. (Directive 92/43/CEE Annexe V), Calendula suffruticosa Vahl subsp. algarbiensis (Boiss.) Nyman ainsi que l'unique station pour le Portugal de Angelica pachycarpa Lange.

La végétation dominante est constituée par des thérophytes et des hémicryptophytes, l'été aucune végétation ne couvre le sol hormis quelques chaméphytes dont Armeria berlengensis Daveau, Asparagus aphyllus L., Euphorbia characias L., Spergularia rupicola Le Jolis, Frankenia laevis L., Lavatera arborea L., Suaeda vera J.F. Gmelin et Tamarix africana Poiret.

Les données sur la Flore de l'archipel proviennent des ouvrages botaniques de Daveau (1884, 1896), Fernández Cigoña (1991), Figueiredo (1972), Franco (1971-1998), Malato-Beliz (1978-1982), Perreira Coutinho (1939), Vasconcelos (1980) et de la consultation des Herbaria de Lisbonne (LISI et LISU). Sont cités quatre témoins d'herbiers qui ne procèdent pas des cueillettes faites par l'auteur, mais de matériel déposé à LISU et dont il n'y a pas de références bibliographiques. La nomenclature suit Flora Europaea (Tutin et al. Eds., 1964-1980), Checklist of Vascular Plants (Smythies, 1984-1986), Flora Iberica (Castroviejo et al. Eds., 1986) et le J. O. N L $206 / 7$ de 22.7.1992 pour les espèces de la
Directive 92/43/CEE.

Les données sur la distribution et l'abondance ont été recueilles par prospection sur le terrain durant la période de 1989-2001, des relevés ont été effectués sur les principales îles de l'archipel (échelle d'abondancedominance de Braun-Blanquet, École Sigmatiste Zurich-Montpellier). La localisation est donnée en coordonnés UTM. Le matériel procédant des cueillettes est déposé à l'Herbarium LISU.

\section{LISTE DES ESPÈCES}

\section{Isoetaceae}

\section{Isoetes histrix Bory}

Dans les replats de granit désagrégé régulièrement inondé et lieux d'écoulement d'eau douce, 29SMD5663, 55-90 m, IV-2001. LISU 173819. Localement abondant Isoetes histrix est l'espèce la plus fréquente du Genre Isoetes dans l'ouest de la Péninsule Ibérique (Prada, 1983; Salvo, Cabezudo \& España, 1984). C'est une espèce caractéristique des communautés thérophytiques régulièrement inondées (Salvo \& Cabezudo, 1984). Sa présence est mentionnée pour les îles Cíes, littoral de Galice (Guitián \& Guitián, 1990).

\section{Papaveraceae}

Fumaria muralis Sonder ex Koch

Sentier du Carreiro dos Cações, 29SMD5663, 50 m, III-1990. LISU 171353. Rare. Espèce de distribution lateatlantique. Au Portugal, cette espèce adventice se localise dans les régions centre, sud et le long du littoral.

\section{Amaranthaceae}

\section{Amaranthus blitoides S. Watson}

Ouest du Carreiro dos Cações, versants rocheux, 29SMD5663, 50 m, VI-1998. LISU 171740. Localement abondant. Il s'agit d'une espèce rudérale dont la présence est récente sur l'île, sa localisation se superpose aux lieux de nidification d'oiseaux maritimes. Espèce allochtone provenant d'Amérique 
du Nord. Cette espèce présente une vaste distribution dans le sud et nord-est de la Péninsule Ibérique, toutefois, la cartographie (UTM 50 x $50 \mathrm{~km}$ ) réalisée par Carretero (1985) ne considère pas le littoral portugais proche de l'archipel.

\section{Portulacaceae}

Montia fontana L. subsp. amporitana Sennen

Versant est de Ilha Velha dans les lieux d'écoulement d'eau douce, 29SMD5663, 55 m, III1990. LISU 171354. Peu abondant. Espèce de distribution méditerranéenne et atlantique, présente dans toute la Péninsule Ibérique (Paiva et al., 1986) tout en étant plus rare dans le sud du Portugal. Espèce hygrophile qui se développe en bordure des cours d'eau, sur les terrains siliceux ou dans des lieux régulièrement inondés. La présence de $M$. fontana subsp. amporitana en zone d'influence maritime a été reportée pour Doñana, dans les dépressions humides (Castroviejo et al., 1979; Cirujano et al., 1992).

\section{Caryophyllaceae}

\section{Cerastium glomeratum Thuill.}

Terrains aux alentours du phare, 29SMD5663, 85 m, III-1990. LISU 171741. Peu abondant. Espèce de distribution subcosmopolite, apparaît dans tout le pays comme rudérale.

\section{Spergula arvensis $\mathrm{L}$.}

Dans la zone du plateau aux alentours du phare, 29SMD5663, 85 m, III-1990. LISU 171742. Peu abondant. Espèce de distribution subcosmopolite. Rudérale qui se développe sur des sols sablonneux, calcifuge.

\section{Stellaria media (L.) Vill.}

Sur le plateau et les coteaux de Ilha Velha, dans les zones de sol profond, 29SMD5663, $65 \mathrm{~m}$, III-1990. LISU 171743. Localement abondant. Espèce des communautés thérophytiques nitrophiles, de distribution subcosmopolite. Se trouve dans tout le Portugal.

\section{Guttiferae}

\section{Hypericum humifusum L.}

Ilha Velha, dans les coteaux d'orientation sud et est, sur sol rocheux, 29SMD5663, 40 m, V-1989. LISU 171744. Peu abondant. Espèce européenne, de distribution ample, plus rare dans le sud du Portugal. Se rattache aux communautés thérophytiques de sols périodiquement inondés, sur terrains siliceux.

\section{Tamaricaceae}

\section{Tamarix africana Poiret}

Plage du Carreiro do Mosteiro, 29SMD5663, 10 m, IV-1991. LISU 171745. Rare, une unique station avec environ six individus. Espèce de l'ouest de la région méditerranéenne. Présent dans le centre et sud du Portugal et dans la vallée du Douro. Croît sur les marges des cours d'eau ainsi que dans les dépressions de sols humides salés ou légèrement salés (Cirujano, 1993).

\section{Rubiaceae}

\section{Rubia peregrina $\mathrm{L}$.}

29SMD56, VI-1924, Palhinha \& Barros, LISU 35700. Espèce de distribution lateméditerannéenne, des forêts sclérophylles, maquis et garrigues.

\section{Brassicaceae}

\section{Coronopus didymus (L.) Sm.}

Dans les dépressions granitiques humides du plateau au nord de Ilha Velha, 29SMD5663, $81 \mathrm{~m}$, VI-98. LISU 171746. Localement abondant. Sa distribution se superpose aux lieux de nidification d'oiseaux maritimes. Espèce allochtone provenant d'Amérique du Sud. Il s'agit d'une espèce des communautés nitrophiles sujettes au piétinement (Costa \& Figuerola, 1983). Sa présence sur l'île résulte d'une situation similaire à celle reportée pour la Corse où son introduction et son expansion sont étroitement liées à la présence de colonies de goélands (Jeanmonod \& Burdet, 1992, 1994).

\section{Brassica oleracea $\mathrm{L}$.}

Sur le plateau au nord de Ilha Velha, en zone de sol profond, 29SMD5663, $70 \mathrm{~m}$, VI-98. LISU 171747. Présence rare, observée depuis 1994. Espèce de distribution méditerranéenne atlantique cultivée dans le monde entier. L'espèce sylvestre est endémique des falaises atlantiques de l'ouest de l'Europe (Gómez Campo, ined.) et la limite 
méridionale de sa distribution se situe dans la Cornisa Cantábrica, au nord d'Espagne (Gómez Campo, 1993). En Galice, cette espèce est considérée comme subspontanée à Lugo (Merino, 1906), plus récemment sa présence a été mentionnée sur les îles Cíes (Rigueiro Rodríguez, 1977). Le niche écologique de cette espèce correspond aux falaises avec nitrification d'origine ornithologique bien que sa présence dans ces lieux puisse être initialement d'origine anthropique (Fernandez Prieto \& Herrera Gallastegui, 1992). Les espèces qui caractérisent ces communautés existent aux Berlengas et se trouvent en expansion; il se peut donc que $B$. oleracea trouve sur l'île les conditions nécessaires à l'établissement d'une population même si sa présence est due initialement à l'introduction fortuite par les oiseaux maritimes.

\section{Euphorbiaceae}

\section{Ricinus communis $\mathrm{L}$.}

Près du village des pécheurs, 29SMD5663, 20 m, VII-1997. LISU 175755. Rare, une unique observation au milieu de Carpobrotus edulis. Espèce allochtone provenant d'Afrique du Sud. Dispersée dans tout le pays en marge de terrains ou de lieux cultivés.

\section{Euphorbia characias L. subsp. characias}

Falaises du Carreiro dos Cações, 29SMD5663, $50 \mathrm{~m}, \mathrm{~V}-1989$. LISU 171355. Confirmation de l'incertitude posée par Daveau sur sa présence. Rare, une unique station peu accessible avec près de 15 individus. Espèce de l'ouest de la région méditerranéenne occidentale. Apparait dans la vallée du Douro et du Sabor, dans le centre et sud-ouest du Portugal. Bien que cette espèce se développe préférentiellement sur les lieux calcicoles (Benedí et al., 1997) elle a été également observée sur granite (Sintra, Santos, LISU 24538) et syénite (Peninha, Rodrigues, LISU 147318).

\section{Euphorbia exigua L.}

Sur le plateau et les coteaux orientés vers le sud et l'est dans les zones de sol peu profond, 29SMD56, 40-80 m, IV-1991. LISU 171748. Peu abondant. Espèce de distribution latéméditerranéenne. Au Portugal, signalée dans les régions nord-est septentrionales, centre et sud. Fait partie des communautés thérophytiques.

\section{Oxalidaceae}

\section{Oxalis pes-caprae $\mathrm{L}$.}

Carreiro do Mosteiro, 29SMD5663, 25 m, III1998. LISU 173820. Distribution localisée, première observation de plusieurs individus en 1999, au milieu de Carpobrotus edulis. Espèce allochtone provenant d'Afrique du Sud. Plante infectante des terrains cultivés.

\section{Oleaceae}

\section{Olea europaea L. var. europaea}

Dans les fentes de la falaise sud de Farilhão da Cova, 29SMD5369, 80 m, V-1991. LISU 171749. Rare, une unique station avec six à huit individus confinés au sommet de l'un des îlots de l'archipel. Les individus croissent contre la paroi, déformés par le vent. Espèce du sud de la région méditerranéenne. Cultivée dans tout le pays.

\section{Solanaceae}

\section{Datura stramonium L.}

Versant nord, 29SMD5663, 10-60 m, VI-1998. LISU 175757. Localement abondant. Première observation sur l'île Berlenga en 1994. Actuellement, s'est répandu avec une certaine abondance sur le versant nord où nidifient les oiseaux maritimes. Espèce allochtone de distribution néotropicale. Sous-spontanée dans presque tout le pays. Rudérale.

\section{Hyoscyamus albus $\mathrm{L}$.}

Dans l'enceinte du fort São João Batista, 29SMD5662, 9 m, V-1989. LISU 171356. Peu abondant. Espèce méditerranéenne. Apparait dans le centre et sud du pays. Plante indicatrice de lieux très nitrophiles, des communautés rudérales des lieux secs et ensoleillés.

\section{Nicotiana glauca R. C. Graham}

Base de la falaise de la plage du Carreiro do Mosteiro, 29SMD5663, 10 m, VI-1998. LISU 175962. Rare, quelques individus isolés. Espèce allochtone provenant d'Amérique du Sud. Rudérale.

\section{Scrophulariaceae}

Bellardia trixago (L.) All. 
Dans la zone du plateau, 29SMD5663, $80 \mathrm{~m}$, V-1989. LISU 171750. Peu abondant. Espèce méditerranéenne. Se trouve dans presque tout le pays. Plante de lieux légèrement nitrophiles, zones rocheuses, sables maritimes et pelouses annuelles.

\section{Asteraceae}

\section{Anthemis arvensis $\mathrm{L}$.}

Sur les coteaux au nord de Ilha Velha, 29SMD5663, $40 \mathrm{~m}, \mathrm{VI}-98$. LISU 171751. Peu abondant et présence irrégulière, observée dès 1995. Distribution dans toute l'Europe (sauf nord), Afrique du Nord, sud-ouest d'Asie. Espèce adventice répandue dans tout le Portugal.

\section{Carduus tenuiflorus Curtis}

Dans la zone du plateau, 29SMD5663, 80m, IV-2001. LISU 173816. Rare. Distribution sud-ouest d'Europe, Afrique du Nord. Rudérale.

Cotula australis (Sieber) Hooker fil.

Sur le plateau au nord de Ilha Velha, 29SMD5663, $80 \mathrm{~m}$, IV-2001. LISU 173817. Localement très abondant. Espèce allochtone provenant de Australie et Nouvelle-Zélande.

\section{Gymnostyles stolonifera (Brot.) Tutin}

Ilha Velha, sur les versants d'orientation sud ef est, sur sol rocheux, 29SMD5663, 40 m, III- 1998. LISU 173821. Rare. Espèce allochtone provenant d'Amérique du Sud.

\section{Helichrysum stoechas (L.) Moench}

Dans l'enceinte du fort São João Batista, 29SMD5662, 9 m, V-1991. LISU 171752. Rare, limité à quelques individus, présence irrégulière. Espèce méditerranéenne. Apparaît dans presque tout le Portugal, dans les terrains arides et sableux.

\section{Xanthium spinosum L.}

29SMD56, VI-1924, Palhinha \& Barros, LISU 37512. Espèce allochtone provenant d'Amérique du Sud. Rudérale de pelouses sèches.

\section{Poaceae}

Aira caryophyllea L. subsp. caryophyllea

Dans la zone du plateau, 29SMD5663, $80 \mathrm{~m}$, V-1989. LISU 171358. Peu abondant dans l'île
Berlenga. Distribution Europe (sauf nord), Afrique septentrionale et méridionale, Asie Mineure. Fréquent dans tout le Portugal. Espèce de pelouses annuelles, sur sols siliceux.

\section{Arundo plinii Turra}

Près de la plage du Mosteiro, 29SMD5663, 10 m, VI-1998. LISU 175756. Rare. Distribution méditerranéenne. Espèce très fréquemment utilisée tout comme Arundo donax L. comme haie coupevent sur les terrains agricoles du littoral. Tous deux peuvent devenir envahissants sur les terrains abandonnés.

\section{Briza maxima L.}

Dans la zone du plateau, 29SMD5663, $80 \mathrm{~m}$, V-1991. LISU 171359. Peu abondant. Distribution lateméditerranéenne. Espèce de pelouses annuelles, sur sols siliceux. Mentionnée pour la province d'Estremadura (Malato-Beliz, 1982).

Chaetopogon fasciculatus (Link) Hayek subsp. prostratus (Hackel \& Lange) Lainz

29SMD56, VIII-1879, Daveau, LISU 3799. Se distribue dans le sud de la région méditerranéenne. Terrains pauvres, sols sablonneux humides.

Hordeum murinum L. subsp. leporinum (Link) Arcangeli

Sur le plateau et les versants sud, 29SMD56, VI-1924, Palhinha \& Barros, LISU 433. Sa présence sur l'île est irrégulière. Peu abondante, $60-80 \mathrm{~m}, \mathrm{~V}$ 1991. Distribution lateméditerranéenne. Espèce rudérale.

\section{Poa infirma Kunth}

Dans les zones de sol rocheux du plateau, 29SMD56, 40-80 m, V-1989. LISU 171753. Localement abondant. Espèce de distribution lateméditerranéenne, répandue sur les terrains pauvres. Espèce tolérante à la salinité, apparaît soit sur le littoral, soit dans les régions endoréiques de climat semiaride de l'intérieur de la Péninsule Ibérique où l'évaporation provoque la salinisation du sol dans les dépressions (Esteve Chueca \& Varo Alcala, 1975; Cirujano, 1981).

Les observations des nouvelles espèces ont été faites sur l'île principale à l'exception 
de Olea europaea var. europaea qui croît dans les fentes des falaises de l'un des îlots. Neuf espèces de cette liste floristique ont une distribution allochtone à la Flore du Portugal et se comportent comme rudérales et en certains cas comme envahissantes. Ce sont des espèces déjà connues depuis le siècle dernier pour la Flore de la Péninsule Ibérique et dont la présence esi mentionnée par Willkomm \& Lange (1861-1880) et Willkomm (1893), hormis Amaranthus blitoides dont la description est postérieure à ces travaux.

Six de ces nouvelles espèces (Fumaria muralis, Cerastium glomeratum, Ricinus communis, Oxalis pes-caprae, Hyoscyamus albus, Nicotiana glauca) se localisent dans des zones à forte fréquentation humaine: Carreiro do Mosteiro (port de pêche, village de pécheurs et plage), Carreiro da Fortaleza (port de plaisance, plage), la zone du phare et les sentiers. Selon Chaloupka \& Domm (1986) l'anthropochorie serait un facteur important de dispersion de diaspores allochtones et ce même facteur serait indépendant de la taille des l'îles.

Amaranthus blitoides, Datura stramonium et Coronopus didymus ont été observés sur les lieux de nidification d'oiseaux maritimes. En effet, l'archipel des Berlengas possède la plus grande colonie résidante de goélands (Larus cachinnans) du littoral portugais. Comme suite aux conditions favorables à son développement, cette colonie a rapidement augmenté. Parallèlement, il s'est vérifié une altération de la végétation avec l'accroissement du nombre et du recouvrement des espèces allochtones. Il semblerait donc exister un rapport entre l'augmentation d'espèces exotiques et la présence d'oiseaux nidifiant, par ailleurs, ce même rapport a été constaté sur d'autres archipels côtiers, pour l'Atlantique nord et la mer Méditerranée.

À partir des observations faites sur l'île de May (Ecosse, UK), Sobey \& Kenworthy (1979) distinguent les activités des oiseaux nidifiant qui peuvent avoir un impact sur la végétation. Ainsi, les perturbations répétées au niveau du sol provoquent l'élimination des espèces pérennes ayant des racines peu profondes et favorisent l'implantation d'annuelles. Par ailleurs, l'apport des déjections conduit à une incrémentation de nutriments disponibles dans le sol qui favorise l'établissement d'une végétation adaptée aux perturbations, composée notamment par des espèces annuelles, rudérales ou maritimes. Hogg et al. (1989) observent sur les îles des Grands Lacs (Lac Huron, Canada) un pourcentage d'espèces exotiques annuelles ou bisannuelles quatre fois plus élevé sur les îlots abritant des colonies d'oiseaux maritimes (Larus delawarensis et L. argentatus) que sur les îles où ceux-ci sont absents. De même, sur les îles Cíes (Galice, Espagne) l'augmentation des communautés d'oiseaux maritimes est à l'origine des altérations de la végétation, avec le remplacement de la végétation naturelle par des communautés de caractère halonitrophile et ornithocoprophile (Guitián \& Guitián, 1989). La même situation s'observe dans la région méditerranéenne, sur les archipels de Riou et du Frioul (Marseille, France). L'augmentation de la densité de goélands a provoqué une prolifération des espèces rudérales et la déstructuration de la végétation originelle. $\mathrm{La}$ comparaison temporelle de la composition floristique de certaines îles indique une grande augmentation du nombre d'espèces rudérales (Vidal, P. et al., 1997; Vidal, E. et al., 1998).

La présence récente sur l'archipel des Berlengas d'espèces rudérales, la variation de leur abondance et de leur distribution sur l'île pourrait donc être une conséquence de l'augmentation de la colonie d'oiseaux maritimes. Néanmoins, selon Sobey \& Kenworthy (1979) si certaines espèces peuvent être considérées nitrophiles ou coprophiles, le concept ne pourrait pas s'étendre à l'ensemble de végétation car les facteurs de l'environnement maritime se superposeraient 
également à ceux de la présence des colonies d'oiseaux.

Par ailleurs, la présence des espèces autochtones Isoetes histrix Bory, Montia fontana L. subsp. amporitana Sennen, Hypericum humifusum L., Aira caryophyllea L. subsp. caryophyllea et Poa infirma Kunth doit être ancienne sur l'île car contrairement aux cas précédents ces espèces ne sont pas liées à des perturbations de l'environnement. Le plateau de l'île Berlenga est fort érodé et les dépressions des replats granitiques se trouvent régulièrement inondées pendant l'hiver. Dans ces dépréssions le granit est fort desagrégé et il se forme une couche de sable sur lequel se développent Isoetes histrix Bory, accompagnée de Juncus bufonius L., Sagina maritima G.Don et Plantago coronopus L., et occasionnellement Poa infirma Kunth.

REMERCIEMENTS. L'auteur remercie l'appui reçu de la Reserva Natural das Berlengas et de la Marine Portugaise (Capitania de Peniche) tout au long de ce travail.

\section{BIBLIOGRAPHIE}

BENEDÍ, C., J. MOLERO, J. SIMON y J. VINCENS -1997- Euphorbia. In Flora lberica, Vol 8. Castroviejo S. et al. Eds. Real Jardín Botánico, CSIC. Madrid.

CARRETERO, J. L. - 1985 - Consideraciones sobre las Amarantáceas Ibéricas. Anales Jard. Bot. Madrid 4(2): 271-286.

CASTROVIEJO, S., E. VALDÉS-BERMEJO, S. RIVAS-MARTÍNEZ y M. COSTA -1979Novedades florísticas de Doñana. Anales Jard. Bot. Madrid 36: 203-244.

CASTROVIEjo, S., M. LAÍNZ, G. LÓPEZ GONZÁLEZ, P. MONTSERRAT, F. MUÑOZ GARMENDIA, J. PAIVA y L. VILLAR Eds. 1986- Flora Iberica, Vols. 1-8. Real Jardín Botánico. Madrid.

CHALOUPKA, M. Y. \& S. B. DOMM - 1986- Role of anthropochory in the invasion of coral cays by alien Flora. Ecology 67(6): 1536-1547.
CIRUJANO, S. - 1981- Las lagunas manchegas y su vegetation. Anales Jard. Bot. Madrid 38(1): 187-232.

CIRUJANO, S. -1993- Tamarix. In Flora Iberica, Vol 3. Castroviejo, S. et al. Eds. Real Jardín Botánico, CSIC. Madrid.

CIRUJANO, S., M. VELAYOS, F. CASTILLA y M. GIL -1992- Criterios botánicos para la valoración de las lagunas y humedales españoles -Península Iberica y las islas Baleares- ICONA CSIC.

COSTA, M. y R. FIGUEROLA - 1983- Contribución al estudio de la clase Polygono-Poetea annaue Rivas Martínez 1975 en Valencia. Anales Jard. Bot. Madrid 40(1): 237-240.

DAVEAU, J. - 1884- Contribuições para a Flora de Portugal Excursion Botanique aux îles Berlengas et Farilhões. Bol. Soc. Brot. 2: 13-31.

DAVEAU, J. - 1896- La Flore Littorale du Portugal. Bull. Herb. Boiss. 4(4): 209-313.

ESTEVE CHUECA, F. y J. VARO ALCALA - 1975Estudio de geobotánica de las comunidades halófilas interiores de la provincia de Granada. Anales Inst. Bot. Cavanilles 32(2): 1351-1373.

FERNANDEZ CIGOÑA, E. -1991- Galegos nas Illas Berlengas: Natureza e Historia. Natureza Galega, Vol. 5. Ed. AGCE. Vigo.

FERNANDEZ PRIETO, J. M. y M. HERRERA GALLASTEGUI -1992- Brassica oleracea L.: Distribución y ecología en las costas atlánticas ibéricas. Lazaroa 13: 121-128.

FIGUEIREDO, J. P. M. - 1972-Contribuição parao estudo de uma Reserva Natural no Arquipélago das Berlengas. Relat. estágio do Curso de Engenheiro Silvicultor. ISA. Lisboa.

FRANCO, J. -1971-1984- Nova Flora de Portugal, Vols. 1-2. Ed. do autor. Lisboa.

FRANCO, J. -1994-1998- Nova Flora de Portugal, Vol. 3 -fasc. I e II- Escolar Editora. Lisboa.

GÓMEZ CAMPO, C. -1993- Brassica. In Flora Iberica, Vol 4. Castroviejo, S. et al. Eds. Real Jardín Botánico, CSIC. Madrid.

GÓMEZ CAMPO, C. -?-ined. Genus Brassica L. EuroMed.

GUITIÁN, J. y P. GUITIÁN -1989- La influencia de las colonias de aves marinas en la vegetacion de los acantilados del Noroeste Ibérico. Bol. Soc. Brot. Sér. 2, 62: 77-86.

GUitián, J. y P. GUITIÁN -1990-El Paisaje vegetal de las islas Cíes. Conselleria de 
Agricultura. Santiago.

HOGG, E., J. MORTON \& J. VENN -1989Biogeography of island floras in the Great Lakes. I. Species richness and composition in relation to gull nesting activities. Can. J. Bot. 67: 961969.

JEANMONOD, D. \& H. M. BURDET Eds. -1992Notes et contributions à la Flore de Corse VIII. Candollea 47(2): 267-318.

JEANMONOD, D. \& H. M. BURDET Eds. -1994Notes et contributions à la Flore de Corse X. Candollea 49(2): 571-612.

JORNAL OFICIAL DAS COMUNIDADES EUROPEIAS N L 206/7 de 22.7. 1992. Directiva 92/43/CEE.

MALATO-BELIZ, J. -1978-1982- Catálogo das plantas infestantes das searas de trigo, Vols. I2 Empresa Pública de Abastecimento de Cereais. Lisboa.

MERINO, B. -1906- Flora descriptiva e ilustrada de Galicia voI. 1. Tipografia Galaica. Santiago.

PAIVA, J., S. CIRUJANO y E. VILLANUEVA 1986- Montia fontana L. (Portulacaceae) en la Península Ibérica. Bol. Soc. Brot. Sér. 2, 59: 321-332.

PERREIRA COUTINHO, X. -1939- Flora de Portugal. $2^{\circ} \mathrm{ed}$. Bertrand. Lisboa.

PRADA, C. -1983- El Genero Isoetes L. en la Peninsula Iberica. Acta Bot. Malacitana 8: 73100.

PUJADAS SALVÁ, A. y A. LORA GONZÁLEZ 1996- El Género Orobanche L. (Orobanchaceae) en la provincia de Almería, SE de España. Acta Bot. Malacitana 21: 199-220.

RIGUEIRO RODRÍGUEZ, A. -1977- Trabajo Botánico sobre las Islas Cíes. ICONA. Madrid.

SALVO, A. E., B. CABEZUDO y L. ESPAÑA 1984- Atlas de la Pteridoflora Iberica y Balear. Acta Bot. Malacitana 9: 105-128.

SALVO, A. E. y B. CABEZUDO -1984- Lista comentada de los Pteridófitos de Andalucía. Acta Bot. Malacitana 9: 133-146.

SER VIÇO METEOROLÓGICO NACIONAL - 1970 O Clima de Portugal. Normais Climatológicas do Continente, Açores e Madeira correspondentes a 1931-1960.

SMYTHIES, B. E. - 1984/1986- Flora of Spain and the Balearic Islands. Checklist of Vascular Plants. Englera 1-3. Botanischer Garten und Bot. Museum. Berlin-Dahlem.
SOBEY, D. \& J. KENWORTHY - 1979- The relationship between herring gulls and the vegetation of their breeding colonies. Jornal of Ecology 67: 469-496.

TAULEIGNE GOMES, C. \& C. LEFÈBVRE. -?Évolution de la végétation de l'île Berlenga Archipel des Berlengas, Estremadura, Portugalsur une période de douze ans. Influence des oiseaux maritimes et possible répercussion sur les taxa endémiques. Bocconea -submitted)

TUTIN, T. G., V. H. HEYWOOD, N. A. BURGES, D. M. MOORE, D. H. VALENTINE, S. M. WALTERS \& D. A. WEBB Eds. -1964-1980Flora Europaea vols. 1-5. Cambridge.

VASCONCELOS, T., J. D. FERNANDES \& F BEIRÃO - 1980- Plantas das Berlengas. Centro Botânica aplicada a agricultura Universidade Técnica. Lisboa.

VIDAL, E., F. MÉDAIL, T. TATONI, P. ROCHE \& P. VIDAL - 1998-Impact of gull colonies on the flora of the Riou archipelago -Mediterranean islands of the south-cast France. Biological Conservation 84(3): 235-243.

VIDAL, P., P. BAYLE, E. VIDAL, F. MÉDAIL \& R. ZOTIER - 1997- Gestion de la faune et de la flore des îles marseillaises. Forêt méditerranéenne 28(1): 44-51

WILLKOMM, M. \& J. LANGE -1861-1880Podromus Florae Hispanicae, Vols. 1-3. Ed. Schweizerbart. Stuttgard.

WILlKomM, M. -1893-Podromi Florae Hispanicae, supplementum. Ed. Schweizerbart. Stuttgard.

Aceptado para su publicación en abril de 2002

Adresse de l'auteur. Museu e Jardim Botânico da Universidade de Lisboa. Rua da Escola Politécnica $\mathrm{n}^{\circ}$ 58, 1250-102 Lisboa. Portugal. E-mail: ctgomes@fc.ul.pt. 